\title{
Cold lesions in inferotemporal cortex produce reversible deficits in learning and retention of visual discriminations
}

\author{
JAMES A. HOREL \\ State University of New York, Upstate Medical Center, Syracuse, New York
}

\begin{abstract}
Cooling devices were implanted bilaterally at four points along the inferotemporal (IT) cortex in three monkeys. Cooling any of the three anterior pairs disrupted performance on a visual memory task (delayed match-to-sample) uniformly over delays of $0,15,30$, and 45 sec. Cooling the most posterior cryode had no effect. Cooling any of the cryodes had no effect on retention of a discrimination between horizontal and vertical stripes. With a focus at the second cryode from anterior, there was significant impairment on learning an object discrimination. With the same focus, a deficit was also found in retention of multiple object discriminations, but there was no deficit in retention of multiple black-white patterns. The anterior two cryodes were then interconnected, as were the posterior two cryodes, so that the anterior or posterior half of IT could be cooled. The animals were tested on retention of a mix of black-white patterns and objects. There were greater deficits on objects anteriorly and patterns posteriorly, but this was marginally below statistically acceptable levels. The four pairs of probes were each cooled separately during tests of retention of discrimination of monkey faces. A depression of scores was produced by all four cryodes, but more at the third from anterior. We suggest that IT is differentiated in the way it processes stimuli, not in the localization of such processes as attention, perception, and learning.
\end{abstract}

In a previous experiment Horel and Pytko (1982) used a cooling device to explore the temporal lobe with small reversible lesions. The behavioral measure was a trialunique delayed match-to-sample (DMS) (Mishkin \& Delacour, 1975; Overman \& Doty, 1980). One temporal lobe was removed, and the cooling probe was moved from place to place in and around the remaining temporal lobe. The only place where a deficit could be consistently produced with this method was in the anterior $9 \mathrm{~mm}$ of the temporal lobe. Subsequently, Horel, Voytko, and Salsbury (1984) permanently implanted two cooling devices bilaterally over the cortex in this area in three monkeys. When only one or the other of these two pairs of cryodes was cooled, a deficit was produced at all delays $(0,15$, 30 , and $45 \mathrm{sec}$ ), but it was worse, approaching chance, at the longest delays. But when both pair of cryodes were cooled, suppressing the function of the entire area that had been identified in the previous experiment (Horel \& Pytko, 1982), performance dropped to chance at all delays and remained poor even with a simultaneous matchto-sample in which all stimuli were present and the animal had to compare them across only the three windows to

This work was supported by NINCDS Grant NS 1829-02. The author wishes to thank Dorothy Joiner for training the animals, Kent Salsbury for constructing the cryodes, Timothy Horel for the equation used in Figure 6, Mary Voytko for critical reading of the manuscript, and Penny Coe for typing the manuscript.

The author's mailing address is: Department of Anatomy, State University of New York, Upstate Medical Center, 766 Irving Avenue, Syracuse, NY 13210. make a correct match. Then the monkeys were tested on learning and retention of the visual discriminations, with the entire polar cortex cooled; a profound deficit on learning the discriminations, but little or no disruption in the performance of discriminations that were learned prior to the application of the cold, was found. The fact that the animals could perform the tasks at normal levels if they were well learned prior to cooling rules out the possibility that either the DMS or the learning deficit was due to some nonspecific effects of the lesions or simple sensory or perceptual disorders.

The severity of the deficit was surprising because previous investigators had expressed some scepticism about participation of the temporal pole in visual function (Brown, 1963; Gross, 1973; Mishkin, 1966); instead, the focus of attention had been upstream in the cortex of the lateral and ventral surface. Certainly, we should expect deficits in the performance of visual tasks with cold lesions in IT. The failure to find such deficits by Horel and Pytko (1982) must be due to the very small lesions that the movable cryoprobe made. In the present experiment, an assembly of four cooling devices that could be implanted along the surface of IT was constructed. These devices were the same size as the cryodes used by Horel et al. (1984) to cover the temporal pole, and should produce much larger lesions than the ones made with the movable cryode, comparable to those made by Iwai and Mishkin (1969). They made small lesions at six different positions along the length of IT and produced deficits in the concurrent learning of several visual discriminations 
with more anterior lesions; deficits in the retention of visual discriminations occurred with more posterior lesions. This qualitative difference between anterior and posterior temporal cortex has subsequently been confirmed by others (Cowey \& Gross, 1970; Sahgal \& Iversen, 1978).

Also, deficits on DMS have been reported to occur with IT lesions (Dean, 1974, 1982; Mishkin \& Oubre, 1976; Sahgal \& Iversen, 1978) and with IT cooling (Fuster, Bauer, \& Jervey, 1981). Thus, our cold lesions should produce deficits on DMS and on learning and retention of visual discriminations.

\section{GENERAL METHODS}

\section{Subjects}

Three experimentally naive female Macaca fasicularis, weighing $2.75,2.95$, and $3.05 \mathrm{~kg}$, were used. During testing, the animals obtained all of their water in the training apparatus for the 5 days per week that they were run. They were weighed each day before testing, and the amount of water they obtained in the apparatus was adjusted for each animal to ensure that they obtained enough to maintain their health but would continue to work steadily for the liquid reward. At the end of the week, they were given limited access to water for $24 \mathrm{~h}$. The water was then removed for an additional $24 \mathrm{~h}$ in preparation for the next week of training.

\section{Training Apparatus}

The apparatus has been described more fully elsewhere (Horel $\&$ Pytko, 1982). The animals were placed in a restraining chair facing three rear-projection screens mounted on a black panel in a sound-attenuating room. The screens were hinged at the top, and a press on a screen actuated a microswitch. The screens measured $8.5 \times 5.5 \mathrm{~cm}$, and there were $5 \mathrm{~cm}$ between the screens. Three Carousel projectors, equipped with 7 -in. lenses with extension tubes, presented the stimuli, on 35-mm slides, to the screens. Shutters controlled the presentation of the stimuli, and the projectors were advanced automatically at the end of a trial. Photocells detected the presence of a hole in the slide mount to inform the computer of the side that would be correct on that trial. An orangeflavored drink, delivered to the mouth by a small tube, was used for reinforcement. The entire apparatus and data collection were controlled by computer. The animal's behavior was monitored during the experiments by a low-light-sensitive television camera with an automatically adjusting iris diaphragm.

\section{Preoperative Training}

The animals were adapted to the apparatus, trained to press the screen for reward, and then trained on delayed match-to-sample. This task will be described in greater detail below. When they achieved a criterion of $90 \%$ correct on this task, they were operated on and cooling devices were implanted over the inferotemporal cortex (IT). Two weeks after surgery, the animals were retested to ensure that performance levels still exceeded $90 \%$ correct.

Postoperatively, seven experiments were performed, using different stimuli and procedures. The procedure for each experiment will be presented together with the results for that experiment.

\section{Cooling Apparatus}

The design and construction of the cryodes is described in detail by Salsbury and Horel (1983). The cryodes consisted of four loops of stainless steel tubing (Figure 1). The 23-ga tubing was $0.64 \mathrm{~mm}$ o.d. and was formed into $3 \times 10 \mathrm{~mm}$ loops and shaped over a casting of the temporal lobes. Above the loop, the tubes were ensheathed in Teflon, which also held a copper/constantan thermocouple. The thermocouple was soldered to the tubing at the point of formation of the loop. The cryode tubing was fastened in place in a stainless

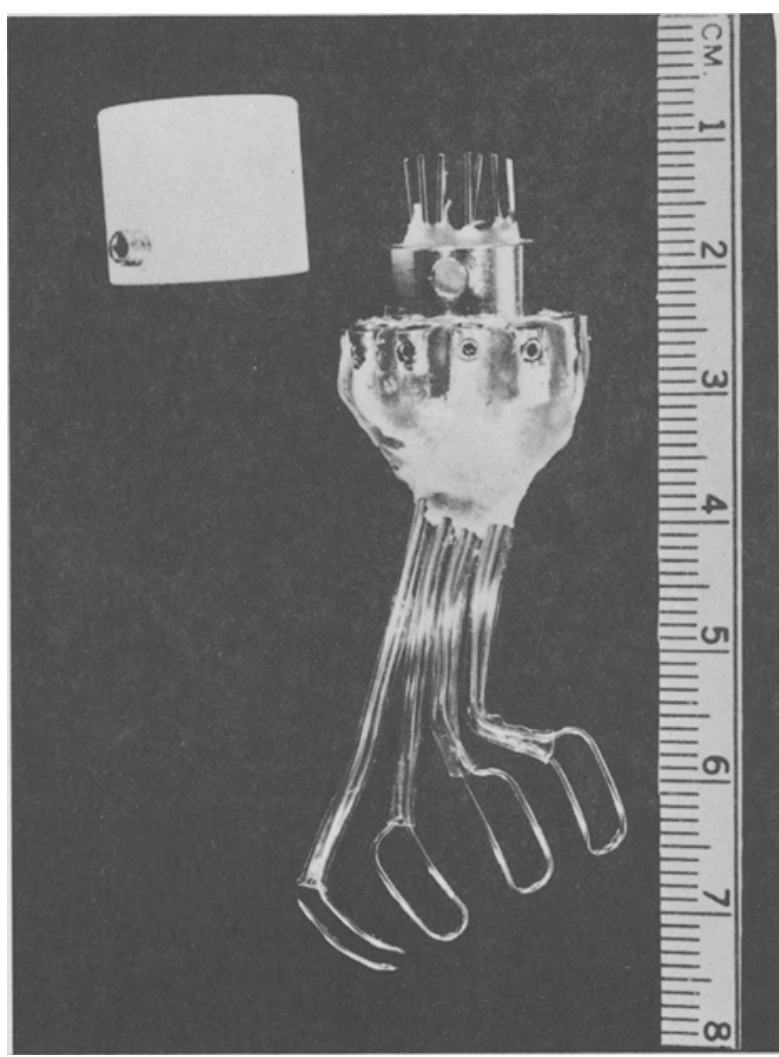

Figure 1. The cryodes before implant. Cap is removed revealing inlet and outlet tubes. When in place, the tubes leading into the cryodes are covered with a thick layer of cranioplastic cement. The cryodes are described in detail in Salsbury and Horel (1983).

steel cylinder $(1.5 \mathrm{~cm}$ i.d.) with a plastic cap. The thermocouple wires were led to connectors outside the cylinder.

The temperature of the cryodes was controlled with cooled methanol. The methanol was pumped from a reservoir through a coil of tubing in a bath of dry ice and methanol. The cooled methanol was conveyed by Teflon tubing to the stainless steel tubes of the cryodes on the animal's head. Outlet tubes led the methanol back to the reservoir. Temperature of the cryodes was monitored with two precision electronic thermometers (Bailey BAT 9 and 12). With thermocouple switchboxes, all eight thermocouples could be monitored using these two thermometers. Two valves located outside of the animal testing room were used to control which of the cryodes on the two sides were cooled. Cryode pairs were ganged together with $\mathrm{T}$ connectors when more than one cryode was cooled on each side. The temperature of the cryodes was set at $0^{\circ} \mathrm{C}$ during experimental trials. All of the control equipment, including the pumps, valves, and thermometers, were outside the animal testing room.

\section{Surgery}

Surgery was conducted with sterile procedures. The animals were anesthetized with sodium pentobarbitol supplemented as needed by a dilute solution of Ketamine iv. A coronal incision was made, and the skull was exposed and cleaned. A block of temporalis muscle was removed where the cooling device was to be mounted. A craniotomy of roughly $7 \times 30 \mathrm{~mm}$ was made over the superior temporal gyrus, and a spatula was inserted between the skull and dura to break adhesions. A gentle rocking motion was used to insert the array of cryodes through the opening and into place over the dura covering the middle and inferior temporal gyri. Four stainless steel 
screws were mounted in the skull around the main cylinder, which was then securely fastened to the screws with cranioplastic cement. The cranioplastic cement was also mounded over the cooling tubes. The skin was sutured and trimmed so as to lie smoothly around the mounting. At the end of the experiment, two of the animals were anesthetized as above and three thermocouples were implanted in each animal for measuring spread of cold in the tissue.

\section{Histology}

Procedure. At the end of the experiment, the animals were given an overdose of sodium pentobarbital and perfused through the abdominal aorta with normal saline followed by $10 \%$ Formalin. The brains were removed with the newly inserted thermocouples in place. Measurements were made of the distance between the thermocouples and the cryodes, and the brains were photographed. Diagrams of the brains were traced from projected images of the photographs, and the placements of the cryodes were drawn on these diagrams, using the indentation they produced as a guide. One of the temporal lobes was blocked, frozen sectioned, and stained with cresyl violet.

Results. The cryodes, held in place by the skull, were tightly pressed to IT. There were clearly visible indentations left by the cryodes (Figure 2), and in some cases the bottom of the loops had pushed slightly into the tissue, creating a fold. The contact with the tissue was poor where the superior temporal gyrus curves into the superior temporal sulcus.

The positions of the cryodes are shown in Figure 3. The position of the posterior bar of the posterior cryode averaged $6.5 \mathrm{~mm}$ anterior to the ascending limb of the inferior occipital sulcus, ranging from 3 to $11.5 \mathrm{~mm}$.

The anterior cryodes were in TG (the temporal pole), sometimes overlapping TE (inferotemporal cortex). The posterior three cryodes were placed on the middle and inferior temporal gyri, called collectively, IT.

There was no evidence that tissue damage was produced by the cold. Occasionally damage was done by cryode placement during surgery, but where this did not occur the cortex looked normal around the indentations produced by the cryode (Figure 4).

Comment. The most anterior cryode appears to be anterior to the most anterior lesions of Iwai and Mishkin (1969); the most posterior placement is at about their lesion II. Their lesions were made in the much larger Macaca mulatta, so these are crude comparisons. However, Desimone and Gross (1979) found the anterior border of the foveal prestriate area and the posterior border of IT to be about $6 \mathrm{~mm}$ anterior to the ascending limb of the inferior occipital sulcus in Macaca fascicularis. Our posterior cryodes are at

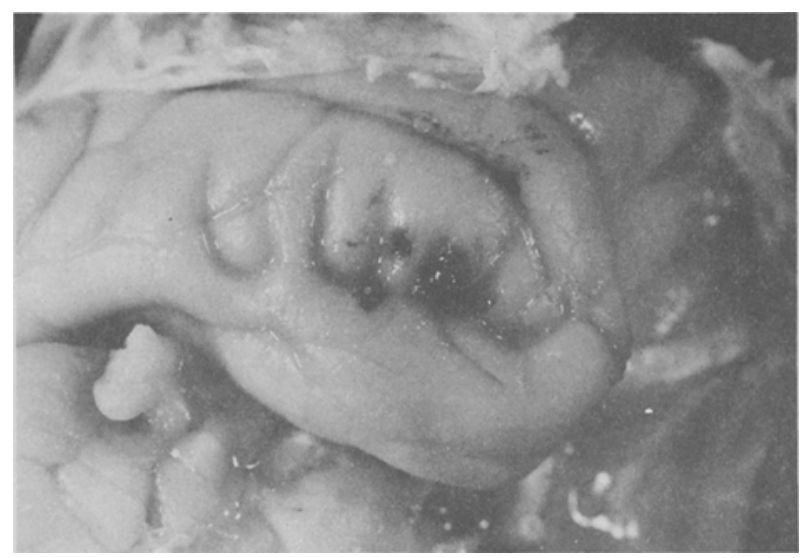

Figure 2. Indentations left by the cryodes. The black spots are the thermocouples that were implanted the day before sacrifice. One of these caused a patch of blood to appear on the surrounding surface, producing the larger black spot.
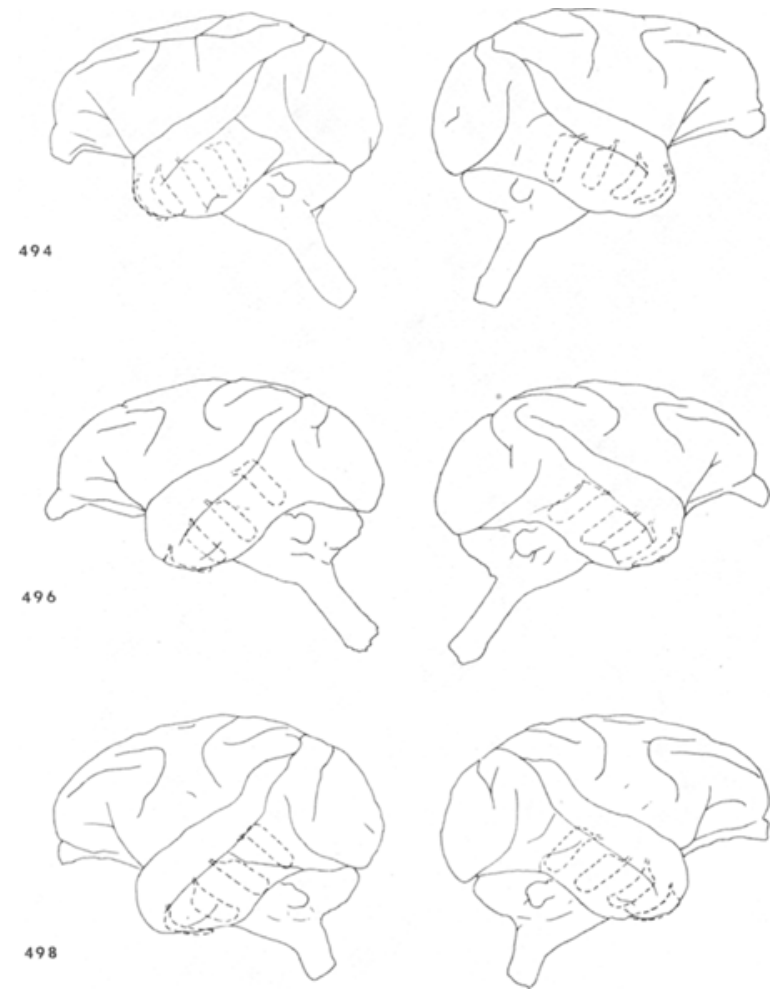

Figure 3. The position of the cryodes indicated with dashed lines.

this border. Therefore, they do not cover the focus of the learning and retention deficits that are found for visual patterns in the foveal prestriate region (area TEO) that lies between the ascending limb of the inferior occipital sulcus and extends $6 \mathrm{~mm}$ rostrally.

The most anterior cryodes lie over the temporal pole (area TG) and border on or overlap anterior IT. The next two cryodes are in the vicinity of the focus of the concurrent learning deficit found by Iwai and Mishkin; the posterior cryodes lie at the posterior border of IT, just anterior to the focus of the visual pattern learning deficit.

\section{Spread of Cold}

Procedure. At the end of the experiments and before the animals were to be killed, six thermocouples were implanted in the cortex next to the cryodes in two animals. The thermocouples were made from 40-ga copper/constantan wire threaded through 22 ga stainless steel tubing and cemented in place with the tip of the thermocouple protruding from the tube. The animals were anesthetized and placed in a stereotaxic instrument; a small skull opening was then made medial to the cylinder that was mounted on the skull. The thermocouples were inserted to the bottom of the skull at a $21^{\circ}$ angle. They were fastened in place with cranioplastic cement adhering to the cement that held the cylinders.

The next day, the animals were placed in their usual position in the apparatus, and the temperature of each cryode was brought to $0^{\circ} \mathrm{C}$, the same temperature that was used during the experiment. The temperature of one thermocouple and one cryode was measured for $6.5 \mathrm{~min}$ using a dual pen chart recorder. This was repeated for all of the thermocouples and all of the cryodes. In the middle of each of these measurements, at $3.5 \mathrm{~min}$, the temperature of all of the thermocouples were sampled.

Results. The temperature of the cryodes reached asymptote at about $1 \mathrm{~min}$ (Figure 5). The tissue temperature dropped more gradually, reaching a low point in about $2 \mathrm{~min}$. When the pumps were turned off, both returned to normal at about the same rate. They 


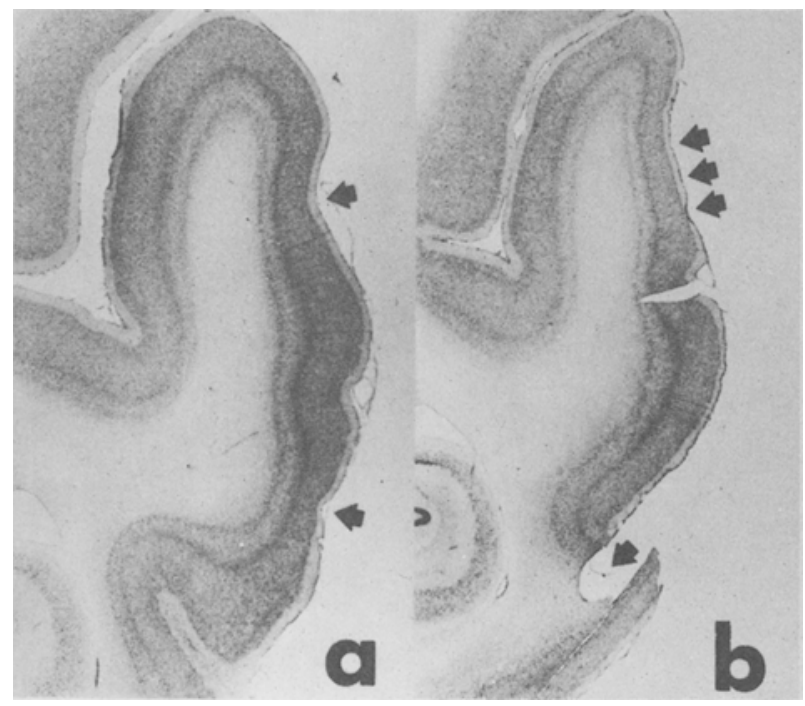

Figure 4. Indentations in IT produced by cryodes are indicated by arrows. In $b$, top, the cut is diagonally through the indentation. In $\mathbf{b}$, bottom, arrow indicates where the bottom of cryode penetrated the tissue.

quickly returned to within about $1^{\circ} \mathrm{C}$ of normal brain temperature (which we find averages at $37.5^{\circ} \mathrm{C}$ ).

Temperature of the cortex rose sharply from the lowest point next to the cryodes, then tapered off to normal body temperature for several millimeters (Figure 6). The temperatures near asymptote were a degree or two below normal body temperature. There are three possible reasons for this: (1) This small amount of cold normally spreads that far in the tissue; (2) the cold spread to adjacent cryodes in the cylinder and these produced a slight cooling of the tissue, or (3) the thermocouples were fastened in the cement with the cryodes, the cold could have been transmitted to the thermocouple by cooling the shaft of the tubing that held them.

The data appear to fit a curve described by the following function:

$$
t=a_{0}+a_{1} e^{a_{2} d},
$$

where $t$ and $d$ are temperature and distance, respectively. The constants were obtained with a least squares procedure. To calculate this, we arbitrarily set 20 points at $37.5^{\circ} \mathrm{C}$ at $6 \mathrm{~cm}$ from the cold. The constants then become:

$$
\mathrm{a}_{0}=37.0, \mathrm{a}_{1}=-32.7 \text { and } \mathrm{a}_{2}=-0.435 \text { (Figure } 6 \text { ). }
$$

Comment. We used unanesthetized animals because we had found (in unpublished work) that brain temperature in anesthetized cats did not return to normal after cooling. A steep isothermic gradiant around a small cold source is consistent with the findings of others, as reviewed by Brooks (1983). A larger cold source appears to produce a greater cold spread (Fuster \& Bauer, 1974). Our measurements were all made with the cold source at $0^{\circ} \mathrm{C}$, but other temperatures should produce differences in the extent of the spread of cold. Bénita and Condé (1972) set a 1.1-mm-diam cryode at $5^{\circ} \mathrm{C}$ and obtained normal body temperature within $3 \mathrm{~mm}$. Using the same cryode as Bénita and Condé set at $-6^{\circ} \mathrm{C}$, Horel and Pytko (1982) estimated, using behavior measures, that functional suppression extended for a radius of $1.6 \mathrm{~mm}$ from the probe.

Synaptic transmission is suppressed in the cortex at $20^{\circ} \mathrm{C}$ (Brooks, 1983; Jasper, Shacter, \& Montplaisir. 1970), which, according to our function, is at $1.5 \mathrm{~mm}$ from the cryode. These are similar to measures we had made previously (unpublished), and is why we constructed the loops to be $3 \mathrm{~mm}$ across and spaced $3 \mathrm{~mm}$ apart. Temperatures must be dropped much lower to disrupt axonal conduction. Bénita and Condé (1972) found that they had to drop their cooling probe to $0^{\circ} \mathrm{C}$ to suppress axonal conduction through the red nucleus. Skinner and Lindsley (1967) blocked conduction in the inferior thalamic peduncle by cooling to $5^{\circ}$ to $10^{\circ} \mathrm{C}$.

\section{EXPERIMENT 1: DELAYED MATCH-TO-SAMPLE}

\section{Procedure}

The stimuli consisted of 740 colored photographs of common objects. An attempt was made to avoid objects that resembled others in the set and to avoid things with which the animal may have had experience, such as food or animals. At the start of a trial, a "sample" stimulus appeared at the center screen and the animal was rewarded for pressing it 10 times. The sample then disappeared, followed by a delay of $0,15,30$, or $45 \mathrm{sec}$. The delays were chosen

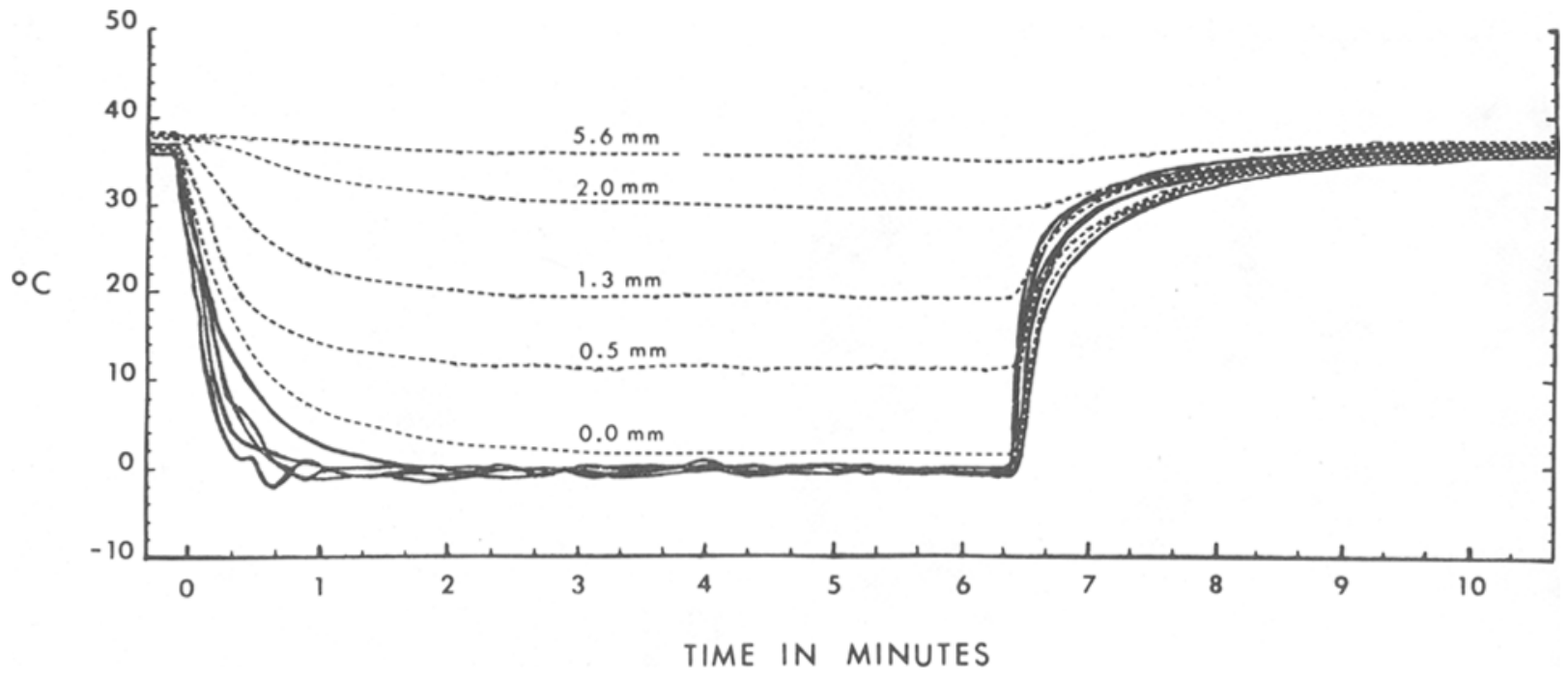

Figure 5. Temperature of cryodes (solid lines) and tissue (dashed lines) as a function of time from onset of pump. 


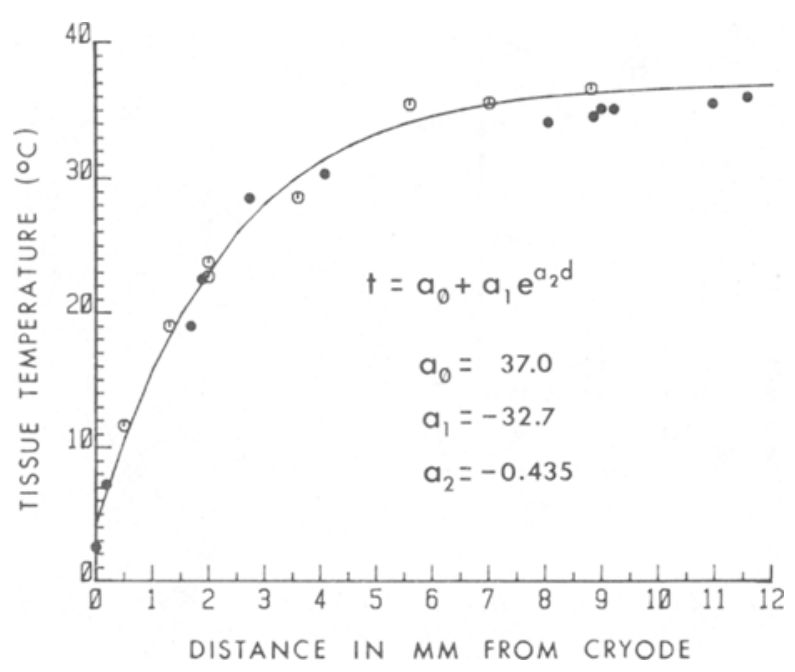

Figure 6. Temperature (t) of tissue as a function of distance (d) from cryode. Cryode temperature was set at $0^{\circ} \mathrm{C}$. The two different symbols represents data from two different animals.

randomly with the restriction that none be repeated until all had been used. After the delay, a stimulus identical to the sample (the "match") appeared at one of the side screens and a nonmatching stimulus appeared at the other side screen. The side on which the match and nonmatch appeared varied according to a Fellows (1967) series. The animals were rewarded for each of two responses to the matching stimulus, which was then extinguished, ending the trial and beginning a $15-\mathrm{sec}$ intertrial interval. A response to the nonmatch was unrewarded, extinguished the stimuli, and initiated a 35-sec intertrial interval. Each stimulus appeared half the time as correct and half as incorrect, and each was paired randomly with different stimuli.

The animals were run in blocks of 20 trials with at least 4 min between each block. Six blocks were run per day for 4 days. A session consisted of two control blocks without cold and four blocks in which one of the four pairs of cryodes was cooled to $0^{\circ} \mathrm{C}$. The order of these conditions was randomized.

Statistical analysis in this and all subsequent experiments was done with analysis of variance (Butler, Kamlet, \& Monty, 1969) with individual comparisons made with the Newman-Keuls test.

\section{Results}

The results are displayed in Figure 7. Cooling produced a depression in performance equally across all delays at the anterior three placements, but not the most posterior placement. In all cases, performance was well above chance levels. There was a significant difference among the experimental and control conditions $[\mathrm{F}(5,10)=9.19$, $\mathrm{p}<.01]$. The effect of delay was not significant $[\mathrm{F}(3,6)$ $=3.77, \mathrm{p}>.05]$, nor was the interaction of delay with the experimental condition $(F=0.6)$. Individual comparisons were made with delays collapsed into one score and the two control scores combined. The anterior three placements were significantly different from the most posterior and from the controls $(p<.05)$. The most posterior placement was not significantly different from controls.

Over the long course of preoperative training, each animal developed its own easily recognizable stereotypic screen-pressing behavior. These behaviors were disrupted by the cold in the early stages of testing, except at the most posterior placement. At first, the animals were slow, seemed confused about how to perform the task and hit the screens in uncharacteristic ways. For example, one animal rested her left hand on the center screen, holding the switch closed, while trying unsuccessfully to actuate the switch with her right hand. The monkeys would often stop and gaze about the room or doze. On the first day, one animal was occasionally so slow, pausing $5 \mathrm{~min}$ or longer, that we advanced the trial to get her to respond. Although these behavioral peculiarities faded toward the end of the DMS experiment, the animals continued to respond in a slow, deliberate manner, particularly when the second or third placements from anterior were cooled. None of these aberrant behaviors appeared while the fourth placement was cooled or during the control trials.

\section{Discussion}

The absence of a delay effect is consistent with the findings of Dean (1974), who used large tissue-removal lesions. We expected our most anterior cryodes to produce defects at the longer delays, as we had found with similar lesions in the temporal pole (Horel et al., 1984). However, in that study, all delays were affected by the cold, but there was a greater effect at the longer delays. The flat curves obtained here suggest some sensory, perceptual, or attentional deficit.

The complete absence of effect from the most posterior placement is evidence that the area cooled by this cryode is not in series with the object-recognition mechanism that has been postulated for IT (Mishkin, 1982). The area just posterior to our most posterior cryode is the foveal prestriate region, or TEO, which is the focus of the visual pattern discrimination deficit (Iwai \& Mishkin, 1969); Kikuchi \& Iwai, 1980). In Mishkin's model, this is the
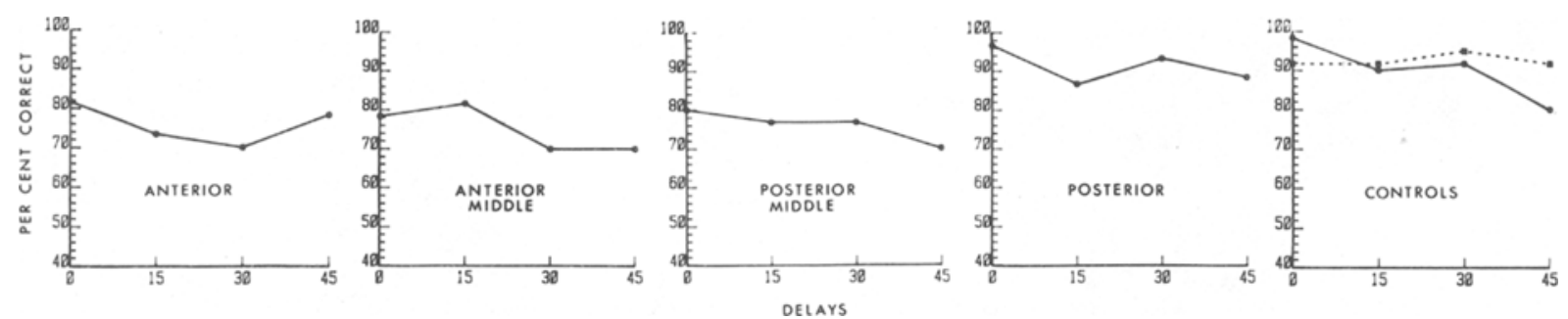

Figure 7. Average performance on delayed match-to-sample during cooling of each of the four pairs of cryodes or during control trials. 
first temporal lobe link in object recognition. The posterior cryode probably did not suppress the function of the entire block of tissue rostral to TEO, but it must have covered a sizable chunk of it. If the model is correct, then the information must skip past this patch of tissue. In any case, the information must get to anterior visual areas from occipital cortex, and this intervening area, cooled by our posterior cryodes, does not appear to be a critical link in this progression of visual function. Indeed, the very high performance levels exhibited while this area was cooled suggest that it may not normally be involved in the task at all.

The initial difficulty these animals experienced in performing the screen-pressing task have not previously been described in studies with IT lesions. This can be accounted for by two factors: (1) Behavioral effects are measured immediately after a cold lesion. With tissue removal lesions, animals are usually not tested for several weeks and immediate postoperative effects, when they have been seen, are dismissed as being the result of surgical trauma. (2) The demands of this screen-pressing task are much greater than the object or plaque displacement that is normally used in WGTA testing.

There are several plausible explanations for the transient screen-pressing difficulty. Perhaps the most obvious derives from the popular theory that IT is involved with object recognition and that lesions impair this function (Mishkin, 1982). The entire apparatus, including the screens the animals press, are objects, and the recognition of these objects, and therefore how to use them, must be impaired if this theory is correct and generalizable to all objects.

Visual functions of animals with IT lesion are typically tested with plaques covering foodwells. The discriminative stimuli adhere to the plaque and often are designs formed from black lines: a visual "pattern discrimination." In the following experiment, we used horizontal and vertical stripes to test retention of a pattern discrimination.

\section{EXPERIMENT 2: RETENTION OF PATTERN DISCRIMINATION}

\section{Procedure}

The stimuli were black and white horizontal and vertical stripes that were $9.4 \mathrm{~mm}$ wide when projected onto the screens. A diffuse light on the center screen initiated a trial, and a response to it extinguished this light and presented the patterns on the side screens, horizontal on one and vertical on the other. Two responses to the horizontal stripes were each rewarded, and a response to the vertical stripes was unrewarded. The animals were run until they achieved $90 \%$ or better on two consecutive blocks of 20 trials. Testing was carried out in the same way as the previous experiment except that there were no delays. The animals were run for 3 days, 120 trials per day in 20-trial blocks.

\section{Results}

The three animals learned this task originally in 40,80 , and 260 trials. Application of the cold did not produce the disruption of screen-pressing behavior that appeared

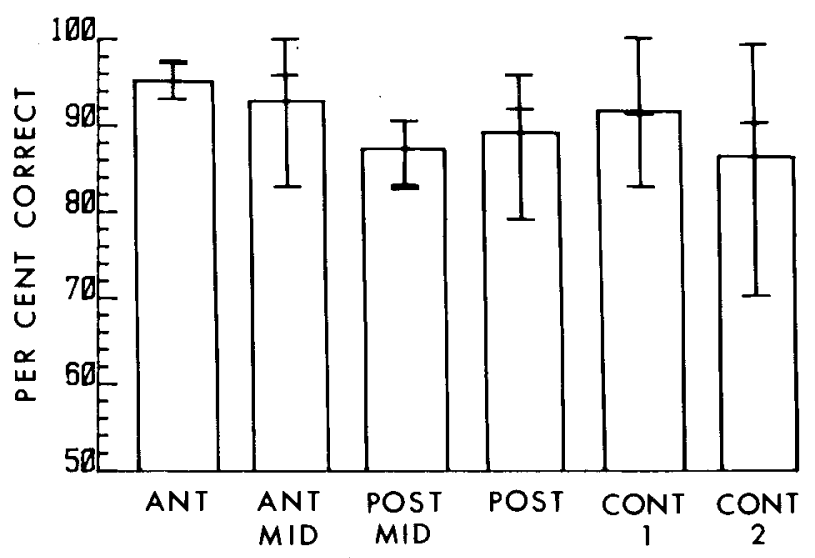

Figure 8. Performance on retention of a horizontal-vertical stripe discrimination while cooling of the anterior pair (ANT), the second pair from anterior (ANT MID), the third pair (POST MID), the posterior pair (POST), or controls (CONT). Each bar represents the average for the three animals.

in the early part of the DMS experiment. It also did not produce a deficit on recall of the visual discrimination (Figure 8; F $=0.937$ ).

\section{Discussion}

This disappointing result must relate to the small size of the cold lesions in this study. When deficits have been found with these stimuli, the lesions are usually much larger. Iwai and Mishkin's (1969) lesions were also small. In Macaca mulatta, they were approximately $5 \mathrm{~mm}$ wide, extending from the superior temporal sulcus to the occipitotemporal sulcus. They obtained weak, but significant, effects from all but the most anterior and posterior placements. However, the focus, where the maximum effect obtains, is a small area, called TEO, bordering posteriorly on the ascending limb of the inferior occipital sulcus (Kikuchi \& Iwai, 1980). It apparently does not include the "middle temporal" area on the posterior bank of the superior temporal sulcus (Kikuchi \& Iwai, 1980; Petrides \& Iversen, 1978). So our failure to obtain an effect is not because we did not cool the superior temporal sulcus. Desimone and Gross (1979) estimated from electrophysiological evidence that its anterior border in Macaca fascicularis was about $6 \mathrm{~mm}$ anterior to the ascending limb of the occipitotemporal sulcus. Our posterior placements bordered on this region; they averaged $6.5 \mathrm{~mm}$ from this sulcus. However, these posterior placements did not extend ventrally as far as we had intended.

Thus, our failure to obtain a deficit on this task can be attributed to the small size of the lesions and their placement anterior to the critical focus in TEO. However, our cryodes were placed directly over the area where Iwai and Mishkin's lesions produced a deficit in learning object discriminations. In their experiment, when several object pairs were presented, one at a time, for learning ("concurrent" design), a deficit appeared at a point we anticipate is under our second cryode from anterior. In 


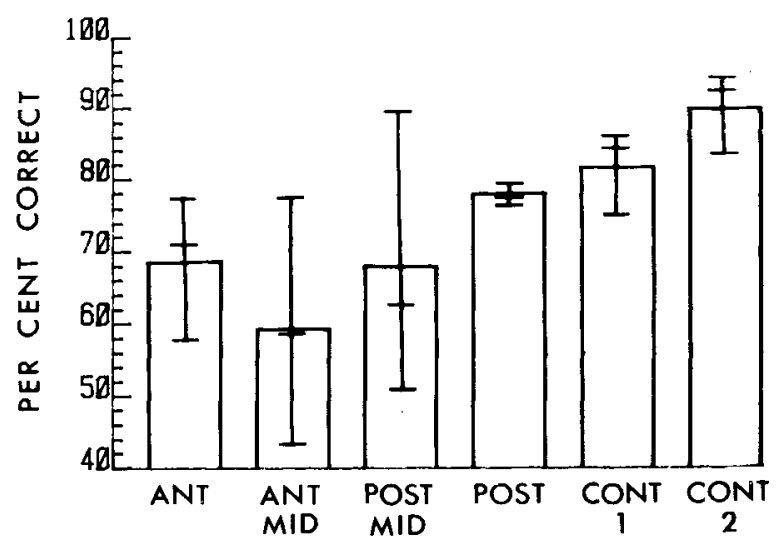

Figure 9. Percent correct in $\mathbf{4 0}$ training trials on learning of object discriminations. Each bar represents the average of the three animals, each with three 40-trial discriminations for each condition.

the next experiment we cooled parts of IT while the animals learned to discriminate between projected photographs of objects.

\section{EXPERIMENT 3: LEARNING PHOTOGRAPHS OF OBJECTS}

\section{Procedure}

A set of new photographs of objects was made for this experiment. Except for the stimuli, the training was the same as in the previous experiment. Two practice days gave us an indication of how long it takes to learn to discriminate a pair of objects. The average was 13.3 trials to a $90 \%$ criterion. Forty trial blocks were run, using one condition and one stimulus pair for a block and changing to a different stimulus and condition for the next block, whether or not the animal had learned the previous stimulus pair. Thus, on experimental blocks, the cold was on throughout the learning of any one pair, and different pairs were used for each of the experimental and control conditions. The animals were run 240 trials per day ( 40 trials $\times 6$ conditions) for 3 days so that three different stimulus pairs were used for each condition. The stimuli were paired randomly and assigned randomly to each animal for each condition.

\section{Results}

There was enormous variability in the rate of learning these object pairs. However, a deficit in learning was concentrated around the second cryode from anterior, as predicted by the Iwai and Mishkin finding (Figure 9). There was a significant main effect $[F(5,10)=3.98$, $p$ $<.05$ ], but the only significant individual comparison was between this second cryode and the best control $(\mathrm{p}$ $<.05$ ).

\section{Discussion}

The deficit in object learning appears to be focused at the same point that Iwai and Mishkin found their concurrent object learning deficit. The concurrent deficit was interpreted as producing an increased load on memory because of the interference associated with learning several discriminations at once. Mishkin (1971) did not find deficits in learning single-object pairs with these lesions; in this, our deficit is more severe than theirs.

In the next experiment, we asked if our lesions would produce deficits in the retention of photographs of objects; recall that we did not find deficits in retention of a horizontal-vertical stripe discrimination. It is simpler to test retention with a concurrent design when each animal is its own control and the same stimulus pairs have to be tested under each condition. On each trial, the animal was presented with a different stimulus pair to recall so that there would be little additional learning for any one pair.

\section{EXPERIMENT 4: CONCURRENT RETENTION OF PHOTOGRAPHS OF OBJECTS}

\section{Procedure}

Twenty new photographs of objects were prepared. They were paired randomly for each animal. The animals were trained on each pair separately, to a criterion of $90 \%$ or better, in two consecutive 20-trial blocks. The pairs were then presented sequentially in random order. The animals received one pair on one trial, a different pair on the next trial, and so on. Two practice days were given at this task before the cold was applied. For the experiment, the animals were run 3 days, 80 trials per day, in 10-trial blocks, alternating experimental (cooling) and control (warm) blocks. They saw a particular pair only once in any 10-trial block. The order of cryode cooling was random, as before.

\section{Results}

The animals took an average of 13.3 trials to reach criterion on the individual stimulus pairs. During cooling, a deficit in retention was found at the same place the deficit in learning was produced (Figure 10). The deficit did not appear to be quite as strong as the learning deficit, and it faded some over the 3 days of testing, but this task produced a stable baseline performance, so there was much less variability. An analysis of variance on the 4 experimental groups and mean of the controls was significant $[\mathrm{F}(4,8)=9.1, \mathrm{p}<.01]$. A Newman-Keuls test showed the second placement from anterior to be significantly different from the controls and from the most

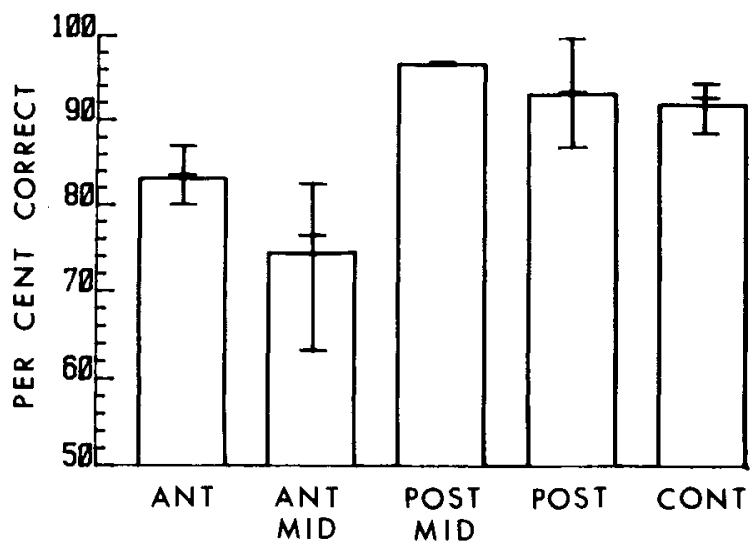

Figure 10. Percent correct on retention of 10 object discriminations presented concurrently. 
posterior placement $(\mathrm{p}<.05)$ and also reliably different from the third placement from anterior $(\mathrm{p}<.01)$.

\section{Discussion}

Retention of a discrimination between photographs of objects was impaired at the same place along IT that we obtained a learning deficit with the same kind of stimuli. But why did we not obtain a retention deficit with our horizontal-vertical stripe discrimination? It may be that the specificity of this area is for a particular type of stimulus: the stripes were of starkly simple, highly redundant components, whereas the objects were highly complex and rich in discriminable elements, with little redundancy. Or, as Cowey and Gross (1970) and Iwai and Mishkin (1969) maintain, it may relate to the special demands of the concurrent task that was used in this experiment. If this latter explanation were correct, then the retention deficit might appear if we used these black-white patterns in a concurrent task.

\section{EXPERIMENT 5: CONCURRENT RETENTION OF BLACK-WHITE PATTERNS}

\section{Procedure}

The stimuli were photographs that projected a black-on-white image. They were geometric shapes or block letters and numbers. The animals were first trained to discriminate 10 pairs of these stimuli, one pair at a time, until they reached $90 \%$ or better in two consecutive 20-trial blocks. After criterion had been reached on all 10 pairs, the stimuli were randomly intermixed and presented one pair at a time as in the previous experiment. This was much more difficult for the animals than was the discrimination of photographs of objects. We had to remove two stimulus pairs and accept an $85 \%$ criterion with 8 concurrent pairs. Reducing the criterion level should increase the probability of obtaining a deficit, because overtraining on the stimuli reduces the chances of obtaining a deficit with IT lesions (Chow \& Survis, 1958; D. R. Meyer, 1958; Orbach \& Fantz, 1958). The animals were then tested in eight-trial blocks so that in any one block the animal saw a particular pair only once. Experimental and control blocks alternated, and the cryode to be cooled on that block varied randomly. The experiment was repeated for three test sessions for 24 trials per experimental condition.

\section{Results}

The animals took an average of 64.2 trials to learn the stimulus pairs. The groups were compared with analysis of variance, using the mean of the control groups as one group. There was no significant difference between the groups (Figure 11; F = 0.41).

\section{Discussion}

The result is consistent with the weak effects obtained by Iwai and Mishkin when they used pattern discriminations instead of objects with anterior lesions. But their weak effects with patterns in contrast to objects was interpreted as being due to the differences in the two tasks they used, not in the stimuli. Our result suggests that the difference between the effects of lesions on retention of objects as opposed to black-white patterns relates to the nature of the stimuli and not the peculiar demands of the concurrent task. The stimuli in the black-white task were designed subjectively to be of the genus of stimuli that

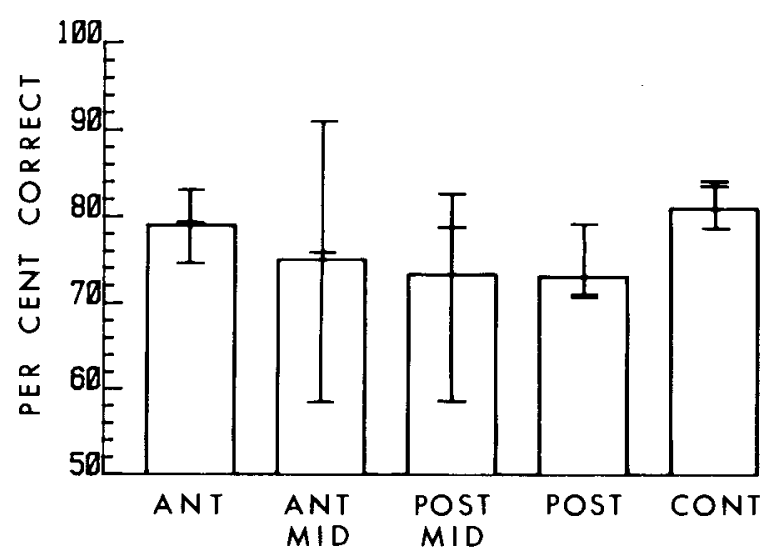

Figure 11. Percent correction on retention of eight black-white patterns presented concurrently.

have been used in the past to demonstrate learning and retention deficits with posterior IT lesions. Perhaps, larger lesions would produce a retention deficit with these stimuli. In the next experiment, we used a mix of objects and black-white patterns and doubled the lesion size. If our hypothesis was correct, anterior lesions would disrupt retention of objects but not of black-white patterns, and we might produce a deficit in pattern discrimination from the posterior lesions, which borders on TEO.

\section{EXPERIMENT 6: CONCURRENT RETENTION OF OBJECTS AND BLACK-WHITE PATTERNS WITH LARGER LESIONS}

\section{Procedure}

Four stimulus pairs were randomly chosen from each of the two previous experiments to make up the eight pairs for this experiment: four colored photographs of objects and four black-white patterns. The pairs were mixed randomly and presented one at a time until they reached a criterion of $80 \%$ correct on both objects and patterns in 1 day.

The two anterior pairs of cryodes were ganged together, as were the two posterior pairs with $T$ connectors so that the anterior two pairs of cryodes were cooled on one side with one pump and on the other side with the other pump. The temperature of the posterior pair was similarly controlled, so that a switch of a valve permitted us to bilaterally control the temperature of the anterior half or the posterior half of IT. With this arrangement, there was always a difference of a few degrees in the temperature of the two cryodes served by one pump that was due to the different flow rates through the cryodes. This was minimized by reversing the leads with some cryodes or by lengthening the leads or covering them with insulating sleeves. With these manipulations, the temperature differences were kept to within $3^{\circ} \mathrm{C}$.

The order of cooling anterior or posterior IT was counterbalanced across animals and days of testing. Experimental and control blocks alternated. Each condition (anterior cool, posterior cool, and control) was run for $\mathbf{1 6}$ trials and was repeated for three test sessions.

\section{Results}

The two control scores were averaged and compared to anterior and posterior cooling (Figure 12). Anterior cooling disrupted performance more on objects than on patterns, whereas posterior cooling disrupted performance 


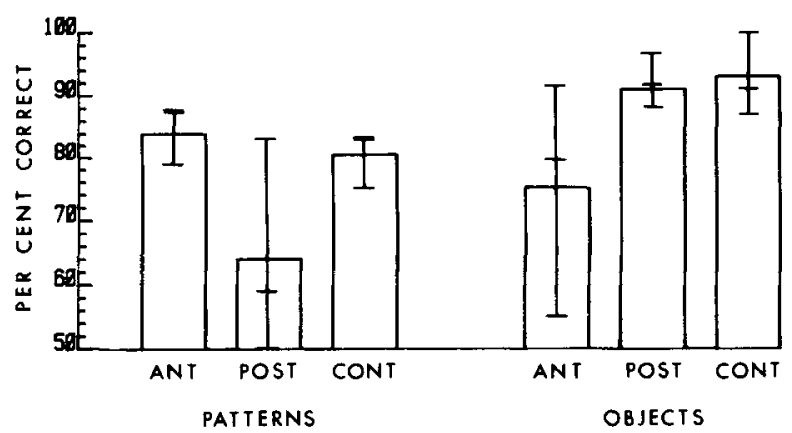

Figure 12. Percent correct while cooling the anterior half or posterior half of IT on retention of a random mix of four object pairs and four pattern pairs presented concurrently.

more on patterns than on objects. A main effect of stimulus difference was significant $[\mathrm{F}(1,2)=25.48, \mathrm{p}<.05]$. However, the experimental condition was not significant $[F(2,4)=1.48, p>.05]$. Of particular interest was the interaction of stimulus type with cooling position; this was marginally below accepted levels for rejecting the null hypothesis $[\mathrm{F}(2,4)=5.22, .05<\mathrm{p}<.1]$.

\section{Discussion}

The results were in the predicted direction and are suggestive of a difference in the stimulus-analyzing properties of anterior and posterior temporal cortex, but they were not strong enough to make the case unambiguously. The effects seem to have been weakened by the use of stimuli with which the animals had already had extensive experience. We had begun to suspect that the effectiveness of the cold in different parts of IT was related to some qualities of the stimulus and had little or nothing to do with variations in the type of task used. The focus of object learning and retention is at our second probe, whereas performance on black-white patterns are affected very little within the reach of any of our cryodes. We searched for a stimulus that might produce a different pattern of deficit, and settled on photographs of monkey faces. This is a complex stimulus, like objects, but with highly redundant elements, like black-white patterns. Some units in IT have been found that appear to be selectively sensitive to faces (Bruce, Desimone, \& Gross, 1981; Leonard, Rolls, Baylis, Wilson, Williams, Griffiths, \& Murzi, 1983).

Another impression that emerged from collecting the data of these experiments was that the sensitivity of the cortex to lesions was not always stable. With repeated testing, some deficits appeared to fade, and a few even appeared to shift from one focus to another over the course of testing. This latter possibility was brought to our attention in Experiment 4, in which a concentration of effect at probe 2 in one animal shifted strongly to probe 1 late in testing. For these reasons, the next experiment involved retention of face discriminations, with the animals' being tested for 10 days.

\section{EXPERIMENT 7: RETENTION OF FACE DISCRIMINATIONS}

\section{Procedure}

Ten colored photographs of Macaca fascicularis faces were made from monkeys in our colony. The animals were photographed faceon in a restraining chair. Two of the animals had cooling devices on their heads; one of these was designated as a negative stimulus (unrewarded) and the other as a positive (rewarded) stimulus; the rest were assigned randomly. We selected photographs of animals with what we judged to be relatively neutral expressions. The monkeys were trained to discriminate the pairs of faces, one at a time, to a criterion of $90 \%$ in two consecutive 20 -trial blocks. The pairs of photographs were then randomly mixed and presented for retention one pair at a time, until a criterion of $85 \%$ in 60 trials was reached. The animals were tested with the cold for 10 days. On each day, all four probe pairs were tested separately in random order with two control blocks randomly interspersed with the experimental blocks. A block consisted of 10 trials.

During the experiment, one of the thermocouples failed in one animal, which forced us to couple the cryode with its mate on the opposite side. This and worsening infections around the cylinders holding the cryodes in two animals forced us to terminate the experiments at the end of this one.

\section{Results}

This discrimination was intermediate in difficulty between the black-white patterns and colored objects. Mean trials to criterion for learning the individual discriminations was 13.3 for objects, 29.3 for faces, 125.3 for the horizontal-vertical pattern, and 68.6 for the black-white patterns learned for the concurrent discrimination. All four cryodes disrupted performances on the face discrimination, and it appeared to remain fairly stable over the 10 days of testing, well above the $50 \%$ chance level (Figure 13). There was a significant overall effect $[F(5,10)$ $=6.12, \mathrm{p}<.01]$. In the individual comparisons (NewmanKeuls), only the third cryode pair from anterior was significantly different from the control groups $(p<.05)$.

\section{Discussion}

One of the reasons for choosing faces as stimuli was the recent evidence that units in the superior temporal sulcus (Perrett, Rolls, \& Caan, 1982) and IT, which projects into that sulcus (Bruce et al., 1981; Leonard et al., 1983), respond selectively to faces. Faces are at least as rich in detail as the objects that were used as stimuli, but, like black-white patterns, they contain many common elements. In difficulty of learning, faces were intermediate between objects and patterns. Although the strongest deficit on faces appeared at the third probe placement from anterior, cooling at all placements produced deficits that were nearly equal.

The focus of deficits for both learning and retention of objects is the same and this is different for black-white patterns and for faces. This confirms the hypothesis that lesion effects in different areas depend upon the nature of the stimuli. This is consistent with findings that severity of the deficit with lesions in IT vary with the parameters of the stimulus (Butter \& Hirzel, 1970; Butter, Mishkin, 


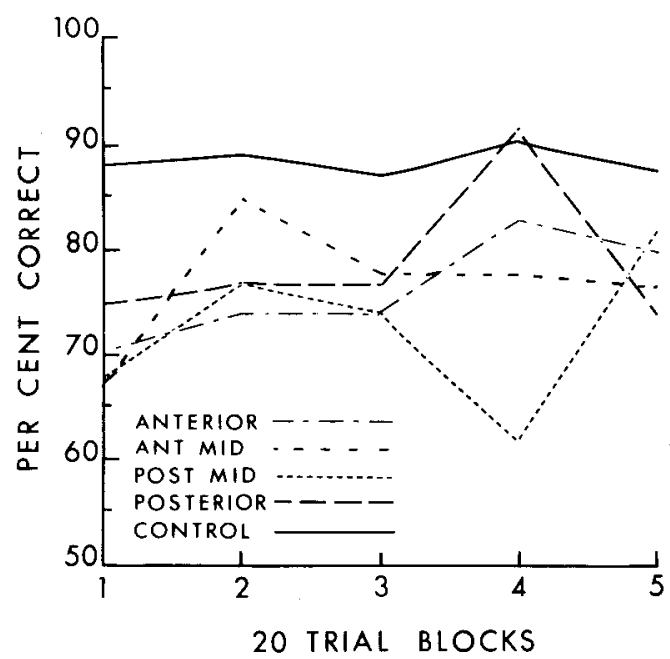

Figure 13. Percent correct in concurrent retention of five pairs of faces as a function of days. Each $\mathbf{2 0}$-trial block is $\mathbf{2}$ days.

\& Rosvold, 1965; D. R. Meyer, 1972; P. M. Meyer \& D. R. Meyer, 1982). It is also consistent with the electrophysiological evidence that single cells within IT fire differentially in response to manipulations of the physical nature of the stimulus, such as outline shape, size, orientation, color, or surface texture, but not to variables relating to the learning task itself (Rolls, Judge, \& Sanghera, 1977). Schwartz, Desimone, Albright, and Gross (1983) found that many IT neurons are selectively sensitive to specific boundary curvature. They were testing a model by Schwartz (1980), which assumes that the neurons in IT code shape on the basis of a "Fourier descriptor." They felt that their model did not account for all of the characteristics of these units, such as responsiveness to three-dimensional objects, color, texture, or complex stimuli such as faces.

From the preceding experiments, it was our impression that there was some instability in the focus of a deficit. This was not confirmed. There appeared to be little drop in error scores over 10 days of testing. There were large daily fluctuations in error scores, but they had no consistent pattern and disappeared when the scores of the animals were averaged. Overtraining provides some protection from the effects of IT lesions (Chow \& Survis, 1958; Orbach \& Fantz, 1958), and some recovery does occur (Chow, 1952). This suggests that some shift in function should occur with lesions and repeated testing. The 10 days of testing in this experiment provided considerable overtraining. It may be that randomly varying the lesion placement forced the animals to constantly vary the part of IT it was using to solve the visual problem. This would produce the wide short-term fluctuations in performance that were seen, but, on average, all areas would show some disruption from the lesion-which is what we found.

\section{GENERAL DISCUSSION}

Application of cold to IT produced the predicted deficits on DMS and learning and retention of visual discriminations, although the deficits were not always in the form, or as severe as, anticipated. We expected to find different lesions along the length of IT to produce different effects. We did find regional effects, but they were neither as crisp nor as stable as we had hoped they would be. The deficits we obtained with essentially the same cryodes in the temporal pole were stronger and more consistent. It is possible that if the cryodes on the lateral surface were oriented to fit the cytoarchitectural divisions that have been established for this area (Seltzer \& Pandya, 1978), we should obtain more consistent and enduring deficits.

The anterior three cryodes produced a deficit on the DMS task, but performance was normal on retention of the horizontal-vertical stripe discrimination over all of the placements. We then attempted to train the animals to discriminate between objects while the cold was applied-a task that had been severely disrupted by the cooling of the temporal pole in Horel et al.'s (1984) experiment. One important difference was that in the earlier study we were cooling two cryodes at once when we produced that deficit, so the lesions then were twice the size that they were for the same task in this experiment. Nonetheless, a focus did appear at the second placement from anterior, a place that looks very like the focus for the concurrent visual learning deficit discovered by Iwai and Mishkin (1969). Thus, we appeared to have a learning deficit without a retention deficit, as in the Iwai and Mishkin finding. Iwai and Mishkin obtained that differential effect with stimuli that were very different-the learning deficit with three-dimensional "junk" objects and the lack of a retention deficit with two-dimensional black-white patterns. In the present experiment, the learning deficit was produced with colored photographs of objects that were designed to resemble, as nearly as photographs can, the junk objects typically used in IT experiments. The retention test was run with black and white horizontal and vertical stripes, designed to be typical of the kind of twodimensional visual patterns that have been used in IT lesion experiments. With our projected stimuli, we obtained qualitatively the same result as that of Iwai and Mishkin: deficits on learning of objects but no retention deficits with the black and white patterns. However, when we used photographs of objects in our retention test, we found a deficit at the same focus as the learning deficit; although it did fade some with repeated testing, it was nonetheless a clear, statistically reliable deficit. Thus, when we used the same stimuli, we got both a learning and a retention deficit from the same place in the temporal lobe.

Perhaps if we tested black-white patterns with the same concurrent retention design that we had used for objects we could elicit a deficit with these stimuli. No deficit appeared. We tried doubling the lesion size to see if we 
would get a stronger effect on retention and a deficit on black-white patterns from this same area. Here we made a mix of objects and black-white patterns and cooled either the anterior half or the posterior half of IT while we tested for retention. Although there did appear to be a difference between anterior and posterior, it did not quite reach statistical reliability; but it is important to notice that the configuration of the result was the same as that of the previous experiments: the anterior lesions produced the most errors with objects, but, with patterns, if there was any deficit at all, it was at the posterior cryodes.

We attempted to change the configuration of the results with a different type of stimulus. Since single cells in IT are particularly responsive to pictures of faces (Bruce et al., 1981; Leonard et al., 1983), we tried retention of a discrimination between photographs of monkey faces. This produced a deficit at all cryodes, but worst at the third from anterior; this is different from objects, for which the deficit was concentrated at the second cryode from anterior, and different from black-white patterns, for which there was no deficit from any of the placements.

These results lead us to suspect that the difference previously discovered between anterior and posterior IT is not a difference between learning and perception or attention, but a difference between the kinds of stimuli that are processed in the temporal lobe. This is not to deny that the anterior temporal lobe is involved in learning and retention, but to suggest that its involvement is with particularly complex stimuli. This interpretation is consistent with observations on single-cell recording in IT: units in IT are sensitive to stimuli with complex shapes and textures, such as hands, faces, or variations in contour (Bruce et al., 1981; Leonard et al., 1983); their discharge rate varies with manipulations in the parameters of the stimuli, but not with manipulations in the parameters of the task (Rolls et al., 1977). The importance of the stimulus configuration and difficulties in attending to specific features of the stimulus by animals with IT lesions has been emphasized by Butter et al. (1965), Butter and Hirzel (1970), D. R. Meyer (1972), and P. M. Meyer and D. R. Meyer (1982). It will take a large number of observations, using different lesion placements with different kinds of stimuli, to determine whether this proposal is correct, but at this point it appears to be at least as likely as the suggestion that anterior temporal cortex is uniquely involved in learning and posterior temporal cortex is uniquely involved in perception.

\section{REFERENCES}

BÉNITA, M., \& CondÉ, H. (1972). Effects of local cooling upon conduction and synaptic transmission. Brain Research, 36, 133-151.

BRooks, V. B. (1983). Study of brain function by local, reversible cooling. Review of Physiological Biochemical Pharmacology, 95, 1-109.

BRowN, T. S. (1963). Olfactory and visual discrimination in the monkey after selective lesions of the temporal lobe. Journal of Comparative and Physiological Psychology, 56, 764-768.

sRuce, C., Desimone, R., \& Gross, C. G. (1981). Visual properties of neurons in a polysensory area in superior temporal sulcus of the macaque. Journal of Neurophysiology, 46, 369-384.
Butler, D. H., Kamlet, A. S., \& Monty, R. A. (1969). A multipurpose analysis of variance FORTRAN IV computer program. Psychonomic Monograph Supplements, 2(16, Whole No. 32), 301-319. ButTER, C. M., \& HirzeL, M. (1970). Impairment in sampling visual stimuli in monkeys with inferotemporal lesions. Physiology \& Behavior, 5, 369-370.

Butter, C. M., Mishkin, M., \& Rosvold, H. E. (1965). Stimulus generalization in monkeys with inferotemporal lesions and lateral occipital lesions. In D. I. Mostofsky (Ed.), Stimulus generalization (pp. 119-133). Stanford: Stanford University Press.

CHOw, K. L. (1952). Conditions influencing the recovery of visual discriminative habits in monkeys following temporal neocortical ablations. Journal of Comparative and Physiological Psychology, 45, 430-437.

CHow, K. L., \& SURvis, J. (1958). Retention of overlearned visual habit after temporal cortical ablation in monkey. A.M.A. Archives of Neurology and Psychiatry, 79, 640-646.

Cowey, A., \& Gross, C. G. (1970). Effects of foveal prestriate and inferotemporal lesions on visual discrimination by rhesus monkeys. Experimental Brain Research, 11, 128-144.

DEAN, P. (1974). The effect of inferotemporal lesions on memory for visual stimuli in rhesus monkeys. Brain Research, 77, 451-469.

DEAN, P. (1982). Visual behavior in monkeys with inferotemporal lesions. In D. J. Ingle, M. A. Goodale, \& R. J. W. Mansfield (Eds.), Analysis of visual behavior (pp. 587-628). Cambridge: MIT Press.

Desimone, R., \& Gross, C. G. (1979). Visual areas in the temporal cortex of the macaque. Brain Research, 178, 363-380.

Fellows, B. J. (1967). Chance stimulus sequences for discrimination tasks. Psychology Bulletin, 67, 87-92.

FUSTER, J. M., \& BAUER, R. H. (1974). Visual short-term memory deficit from hypothermia of frontal cortex. Brain Research, 81, 393-400.

Fuster, J. M., Bauer, R. H., \& JeRVey, J. P. (1981). Effects of cooling inferotemporal cortex on performance of visual tasks. Experimental Neurology, 71, 398-409.

Gross, C. G. (1973). Visual functions of inferotemporal cortex. In R. Jung (Ed.), Handbook of sensory physiology (Vol. 7, Part 38, pp. 451482). Berlin: Springer.

Horel, J. A., \& PytKo, D. E. (1982). Behavioral effect of local cooling in temporal lobe of monkeys. Journal of Neurophysiology, 47, $11-22$.

Horel, J. A., Voytro, M. L., \& SalSBury, K. G. (1984). Visual learning suppressed by cooling the temporal pole. Behavioral Neuroscience, 98, 310-324.

IWAI, E., \& MISHKIN, M. (1969). Two visual foci in the temporal lobe of monkeys. In Neurophysiological basis of learning and behavior. Osaka: Osaka University Press.

Jasper, H., Shacter, D. G., \& Montplaisir, J. (1970). The effects of local cooling upon spontaneous and evoked electrical activity of cerebral cortex. Canadian Journal of Physiological Pharmacology, 48, 640-652.

KIKUCHI, R., \& IWAI, E. (1980). The locus of the posterior subdivision of the inferotemporal learning area in the monkey. Brain Research, 198, 347-360.

Leonard, C. M., Rolls, E. T., Baylis, G. C., Wilson, F. A. W., Williams, G. V., Griffiths, C., \& Murzi, E. (1983). Response properties and distribution of neurons which respond to faces in the monkey. Society of Neuroscience Abstract, 9, 958.

MEYER, D. R. (1958). Some psychological determinants of sparing and loss following damage to the brain. In H. F. Harlow \& C. N. Woolsey (Eds.), Biological and biochemical basis of behavior (pp. 173192). Madison: University of Wisconsin Press.

Meyer, D. R. (1972). Some features of the dorsolateral frontal and inferotemporal syndromes in monkeys. Acta Neurobiologica Experientia, 32, 235-260.

Meyer, P. M., \& Meyer, D. R. (1982). Memory remembering and amnesia. In R. L. Isaacson \& N. E. Spear (Eds.), The expression of knowledge (pp. 179-212). New York: Plenum Press.

MishKIN, M. (1966). Visual mechanisms beyond the striate cortex. In R. W. Russell (Ed.), Frontiers in physiological psychology (pp. 93119). New York: Academic Press. 
Mishrin, M. (1971). Cortical visual areas and their interactions. In A G. Karczmar (Ed.), The brain and human behavior (pp. 187-208). Berlin: Springer.

Mishkin, M. (1982). A memory system in the monkey. Philosophical Transactions of the Royal Society, London, B, 298, 85-95.

MishKin, M., \& Delacour, J. (1975). An analysis of short-term visual memory in monkeys. Journal of Experimental Psychology: Animal Behavior Processes, 1, 326-334.

Mishkin, M., \& OUbre, J. L. (1976). Dissociation of deficits on visual memory tasks after inferior teniporal and amygdala lesions in monkeys. Society for Neuroscience Abstracts, 2, 1127.

OrBACH, J., \& FANTZ, R. L. (1958). Differential effects of temporal neocortical resections on overtrained and non-overtrained visual habits in monkeys. Journal of Comparative and Physiological Psychology, 51, 126-129.

Overman, W. H., \& Doty, R. W. (1980). Prolonged visual memory in macaques and man. Neuroscience, 5, 1825-1831.

Perretr, D. I., Rolls, E. T., \& CAAN, W. (1982). Visual neurons responsive to faces in monkey temporal cortex. Experimental Brain Research, 47, 329-342.

Petrides, M. \& IVERSEN, S. D. (1978). The effect of selective anterior and posterior association cortex lesions in the monkey on performance of a visual-auditory compound discrimination task. Neuropsychologia, 16, 527-537.
Rolls, E. T., Judge, S. J., \& SANghera, M. K. (1977). Activity of neurons in the inferotemporal cortex of the alert monkey. Brain Research, 130, 229-238.

SAHGAL, A., \& IVERSEN, S. D. (1978). The effects of foveal prestriate and inferotemporal lesions on matching to sample behavior in monkeys. Neuropsychologia, 16, 391-406.

Salsbury, K. G., \& Horel, J. A. (1983). A cryogenic implant for producing reversible functional brain lesions. Behavior Research Methods \& Instrumentation, 15, 433-436.

SchwaRTz, E. L. (1980). Computational anatomy and functional architecture of striate cortex: A spatial mapping approach to perceptual coding. Vision Research, 20, 645-669.

Schwartz, E. L., Desimone, R., Albright, T. D., \& Gross, C. G. (1983). Shape recognition and inferotemporal neurons. Proceedings of the National Academy of Science, 80, 5776-5778.

Seltzer, B., \& PANDYA, D. N. (1978). Afferent cortical connections and architectonics of the superior temporal sulcus and surrounding cortex in the rhesus monkey. Brain Research, 149, 1-24.

SKINNER, J. E., \& LiNDSLEY, D. B. (1967). Electrophysiological and behavioral effects of blockade of the nonspecific thalamo-cortical system. Brain Research, 6, 95-118.

(Manuscript received May 23, 1984; revision accepted for publication September 18, 1984.) 